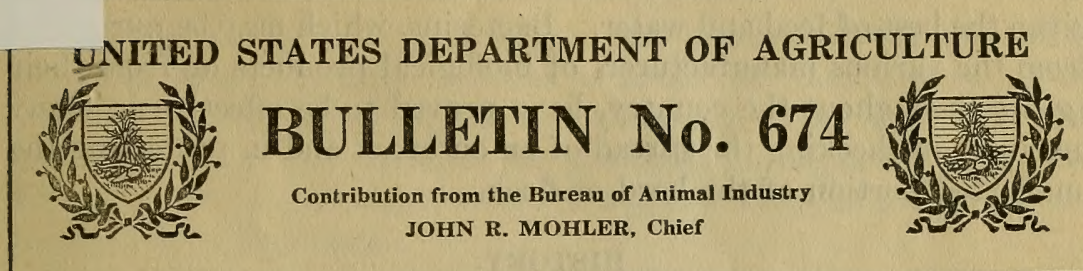

Washington, D. C.

PROFESSIONAL PAPER

May 15, 1918

\title{
HEMORRHAGIC SEPTICEMIA.
}

\author{
By Henry J. Washburn, \\ Senior Pathologist, Pathological Division.
}

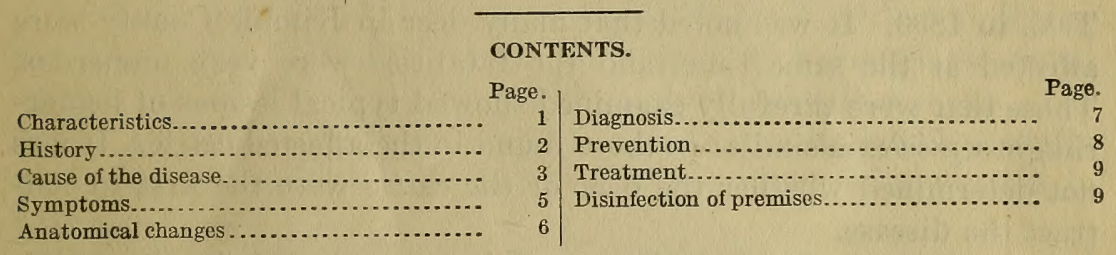

\section{CHARACTERISTICS.}

Hemorrhagic septicemia is an infectious disease, attended with a very high mortality, which attacks various species of animals, especially cattle, sheep, and swine. Young animals are more susceptible to the disease than older ones, and those that are thin and poorly nourished are most liable to be affected.

The disease is a septicemia, or poisoning of the blood, wherefore it often runs a short course and the affected animal quickly dies. In suddenness of attack and high mortality at the beginning of an outbreak there is great resemblance to anthrax.

In hogs the disease is known as swine plague. The acute form is usually fatal to hogs within a few hours from the appearance of the first symptoms. In chronic cases the affected hogs gradually become weaker and thinner and may linger for several weeks.

Fowl cholera represents the avian form of hemorrhagic septicemia, and its attacks sometimes cause great losses. Pigeons and geese are susceptible to hemorrhagic septicemia and the introduction of the infection into flocks of birds of those kinds may lead to numerous. fatalities.

The disease usually results in death so quickly at the beginning of an outbreak that no forms of treatment have time to become effective. The apparently healthy animais should be separated from the diseased and placed in clean, uninfected quarters, where they should be $46449^{\circ}-$ Bull. $674-18$ 
given the best of feed and water. Bacterins, which may be purchased from the various manufacturers of biological products or from their agents throughout the country, have proved to be effective in many instances in checking the spread of an outbreak and in protecting the unaffected portion of the herd or flock.

\section{HISTORY.}

The disease occurred many years ago in this country in the form of swine plague. In 1885, through bacteriological studies that were carried on in the Bureau of Animal Industry, the Bacillus bipotaris suisepticus was identified as the cause of swine plague in the United States, and at the same time the disease was identified as the "Schweineseuche" of German writers.

Hemorrhagic septicemia appeared among cattle in Kimble County, Tex., in 1896. It was noted that many deer in Kimble County were affected at the same time, and the fatalities were very numerous. Those that were carefully examined showed typical lesions of hemorrhagic septicemia similar to those found in the affected cattle. It was not determined whether the deer or the cattle were the first to contract the disease.

In 1898 cattle owners in Tennessee lost many of their young animals from hemorrhagic septicemia. In this instance the infection appeared to be spread over a considerable area, and the disease seemed inclined to assume an unusually chronic character.

Several outbreaks occurred among cattle in Minnesota in 1900. The cases were carefully studied by Brimhall and Wilson, and the cultural characteristics of the microorganism that was isolated from the affected cattle were definitely established.

A year or two later some young cattle were affected while on a hilly pasture in central Virginia, where they had been feeding about one week when the disease appeared. Removing all the drove to a lowland pasture stopped the losses promptly. It was reported by the owner that it was necessary to abandon the mountain lot as a cattle pasture, as the disease recurred each spring if cattle were allowed to run over the infected areas.

During the month of December, 1911, the Department of Agriculture was notified by the Department of the Interior that a fatal disease had appeared among the buffaloes in the Yellowstone National Park, young animals being most susceptible. In all, 22 buffaloes died between December 3 and December 15. Examination of specimens of affected organs at the Washington laboratory of the bureau revealed an infection with the specific microorganism of hemorrhagic septicemia, and pure cultures of those bacilli were obtained. In order to prevent a recurrence of the infection in the 
spring the entire herd of buffaloes was vaccinated with material prepared from the pure cultures obtained in Washington. Two vaccines of different strengths were prepared. The vaccine for the first inoculation was prepared by growing the organism 5 days at $42.5^{\circ} \mathrm{C}$. $\left(108^{\circ} \mathrm{F}\right.$. $)$, while the second was cultivated at the same temperature for only 2 days.

This experiment was probably the first instance in which autogenic vaccines were used in the United States for the prevention of hemorrhagic septicemia, and its success was so marked that similar steps were taken afterwards to check outbreaks among cattle, sheep, and goats, so that at the present time bacterins for the inoculation of infected herds and flocks are prepared by several firms which manufacture biological products and may be purchased in all parts of the country.

Anthrax appeared in several counties in Texas in 1913, and during the investigation of the outbreak two cases of hemorrhagic septicemia were discovered, one in a horse and the other in a sheep. The organisms recovered were identical with the bacilli, which recently have been recovered from outbreaks of hemorrhagic septicemia in cattle and goats.

During the fall and winter of 1915-16 many cases were reported to the Bureau of Animal Industry in which young stock cattle that had been purchased in carload lots at some of the large stockyards had developed hemorrhagic septicemia within a few days after their arrival. At the same time two flocks of sheep and one of goats were found to be affected with the disease. Cultures of either the hemorrhagic septicemia group of organisms or cultures closely related to the colon or paratyphoid B group were recovered from these cases.

\section{CAUSE OF THE DISEASE.}

The organism of hemorrhagic septicemia, the Bacillus bipolaris septicus, belongs to the group in which the bacilli of chicken cholera, swine plague, and rabbit septicemia are to be found. This group is known also by the name Pasteurella. The individual organism is an ovoid, nonmotile, polar-staining bacterium with rounded ends, $1 / 38000$ of an inch wide by $1 / 20000$ of an inch long, sometimes seen in pairs or chains, but usually appearing singly. It stains readily with the stains usually used in bacteriological work, and in many cases shows deep staining at each end, while the middle of the bacillus shows but little coloring. It forms no gas and quickly destroys itself while growing on solid culture media by the development of acids.

The organism may be cultivated readily in bouillon and on agar and gelatin. The reaction of the medium should be slightly alkalin or neutral. It does not liquefy gelatin, coagulate milk, or produce indol. 
The bipolar staining property of the organism may be demonstrated readily in preparations made from the tissues or body fluids (kidneys, blood, etc.), cultures of the organism usually giving less pronounced results in this respect.

Attempts have been made during an outbreak of hemorrhagic septicemia among cattle to transfer the disease from affected to healthy animals by means of rubbing saliva from diseased cattle into the mouths of healthy susceptible animals, and by injecting serum from the blood of a diseased yearling beneath the skin of susceptible young cattle, but without success. Many attempts to infect hogs by natural means from diseased cattle also have failed. In one instance, however, a colt that fed from the rack with a number of diseased sheep contracted the disease and died.

The spread of the disease seems to depend nearly as much upon the condition and susceptibility of the animal as upon the contagious nature of the disease, as thin, poorly nourished young stock most frequently become infected and die of septicemia.

In a number of outbreaks of a disease resembling hemorrhagic septicemia in all its manifestations and anatomical changes an organism which differs in cultural characteristics from the true $B . b i$ polaris septicus has been recovered. This organism proves to be virulent for experimental animals, (rabbits and guinea pigs), producing in them changes suggestive of hemorrhagic septicemia. In preparations from affected tissue or body fluids the organism stains bipolar, and usually occurs singly or occasionally in pairs. It differs from the true $B$. bipolaris septicus in that it appears slightly larger, possesses a sluggish motility, and produces gas in sugar media. In its cultural characteristics it corresponds in most instances to bacteria of the colon group, although some of the characteristics possessed by the paratyphoid B group have been noted.

Bipolar ovoid bacilli which closely resemble the organism of hemorrhagic septicemia are widely distributed in nature. They have been found in the soil, upon various plants, in stagnant water, and upon the moist nasal membranes of normal calves and hogs. In several instances these harmless organisms have been so increased in virulence by passing through animals that they finally proved to be fatal when injected into pigs, and in those instances the tissue changes, which were found at the autopsy of the pig, were similar to those found in swine that had died from swine plague.

It is thought by some writers that after the organisms have become virulent enough to cause an outbreak among animals, they will iater, after that infection has been overcome, return to their previous harmless stage. The increased virulence which is made evident by an attack of several animals of a single species appears to be effective only in animals of that particular species, and the disease does not 
spread to individuals of other species. For example, hogs and sheep that are pastured with a drove of cattle in which several deaths occur from hemorrhagic septicemia usually remain unaffected, although on another farm the sheep or the hogs alone may contract the disease and all the cattle escape. If any exceptions to this rule occur they are extremely rare.

\section{SYMPTOMS.}

In cattle the disease develops very rapidly, running a course of from 1 to 8 days. There is usually a steady elevation of body temperature until from $104^{\circ}$ to $107^{\circ} \mathrm{F}$. $\left(40^{\circ}\right.$ to $41.67^{\circ}$ C. $)$ is reached. The animal refuses its feed. Swelling may appear beneath the skin of the head, throat, or dewlap. These enlargements are somewhat soft and pit on pressure. The tongue is often extensively swollen, and the animal drools and slobbers because of the irritation to its tongue and throat. There may be difficulty in breathing, depending on the degree of involvement of the air passages and of the lungs. Occasional coughing may occur. Muscular trembling may be evident. There may be a blood-stained discharge from the nostrils, and strings of mueus may hang from the mouth. Examination of the nostrils often reveals the presence of many small hemorrhages just beneath their lining membranes. The eyelids become highly inflamed and as a result tears flow down the cheeks.

There is an intestinal form in which the changes are chiefly found in the abdominal cavity, or the intestinal form may develop after the disease has appeared in the lungs. The stomach, intestines, and kidneys and the lymph glands belonging to them become studded with hemorrhages of various sizes, and the intestines become intensely inflamed. The consequence of the developments is that diarrhea sets in, accompanied with the passage of shreds of mucus and of bloody feces. The intestinal form is rare, as most cases show severe involvement of the lungs and the symptoms of croupous pneumonia. The animals may stand with their forelegs wide apart in order to breathe more freely. They lose flesh very rapidly when affected with hemorrhagic septicemia, their abdomens become "tucked up," and the eyes quickly become sunken. A staggering gait, caused by the extreme weakness of the patient, is sometimes noticed.

A disease has been described under the name of septic pleuropneumonia of calves, which is a form of hemorrhagic septicemia, and is caused by the Bacillus bipolaris vitulisepticus. The symptoms shown by the affected calves are quite characteristic of hemorrhagic septicemia, and the post-mortem findings are also those found in that disease. 
In sheep, young animals which have just been weaned are found to be most susceptible, the disease manifesting itself in an acute form. There is marked depression, high temperature, labored respiration, loss of appetite, muscular trembling, and frequently colicky pains. A subacute and a chronic form of the disease are also recognized, the latter affecting principally older sheep. Aside from the febrile changes, weakness, etc., there is noted in the subacute form a discharge from the eyes and nose which at first is serous, later becoming purulent. There may be also pulmonary impairment (pneumonia), or there may be evidence of enteritis. Occasionally the symptoms subside only to return in a more chronic form, which manifests itself principally as a chronic affection of the lungs, with gradual emaciation of the animal. Sometimes the joints are involved, swelling of the knee joints being noted in some cases.

In swine the disease sometimes manifests itself in a peracute form, the animal showing symptoms of a general septicemic condition. Red spots may be noted on various parts of the body, especially around the ears and on the neck and rump. When affected with this form of the disease the animal usually dies within a few hours after the first manifestations of symptoms. In acute swine plague the disease usually occurs as a necrotic pleuropneumonia. There is labored respiration, dry, spasmodic cough, a slimy discharge from the nose, sometimes a purulent conjunctivitis, cyanotic membranes, constipation followed by diarrhea, the feces sometimes containing blood. The animal becomes greatly emaciated and usually dies in from 1 to 2 weeks. Sometimes the acute form changes into the chronic type, in which case the acute symptoms subside, and the cough and evidence of pulmonary involvement continue for a considerable time. Progressive emaciation occurs and a chronic inflammation of the joints may develop. The animals die in from 3 to 6 weeks from complete exhaustion. Sometimes, however, the lung foci may become encapsulated, and the animals may even be fattened in spite of the condition of the lungs.

\section{ANATOMICAL CHANGES.}

Swellings will be found in the subcutaneous tissues. If these enlargements are examined they are found to consist of collections of jellylike material tinged with blood. Occasionally they are limited to a single shoulder or flank, when they may be mistaken for blackleg. The lymph glands are enlarged and are injected with blood. The mucous membranes which line the respiratory tract are similarly affected. False membranes composed of fibrinous exudate may develop in the throat. The spleen remains normal. Hemorrhages are constant in the connective tissues around the kidneys and within the walls of the intestines. 
In the pectoral form the lungs are darkened in color with their fibrous tissues much thickened from the collection of bloody serum in their meshes. The diaphragm, heart sac, and heart walls show numerous bloody points and larger hemorrhages.

In the intestinal form hemorrhages into the intestines will be present and sloughing of the lining of the intestinal wall will be observed, as a result of which the intestinal contents will be wrapped in a covering of bloody mucus.

In acute forms the animals may die suddenly and the changes that are present in such cases will not be very marked. Microscopic examination of the body fluids in these cases will demonstrate the presence of numerous specific coccobacilli.

\section{DIAGNOSIS.}

Because of their acute course, high fever, and rapid termination in death, some difficulty may be experienced in distinguishing anthrax, malignant edema, and blackleg from hemorrhagic septicemia. The differentiation of hog cholera from hemorrhagic septicemia of swine also presents many puzzling points.

There may be edematous swelling of the throat or neck in eitheranthrax or hemorrhagic septicemia. An examination of the spleen of the affected animal will give a conclusive diagnosis, for the spleen of an animal dead from anthrax nearly always becomes acutely swollen and its pulp becomes softened. Small hemorrhages are usually present in the kidney fat in cases of hemorrhagic septicemia. Bacteriological examination will demonstrate quickly the presence of the specific organisms of anthrax or of hemorrhagic septicemia, and a test should be applied in all cases in which doubt exists. The value of a definite diagnosis will be recognized when the lasting nature of an anthrax infection and the more transitory character of an outbreak of hemorrhagic septicemia are considered.

Blackleg and malignant edema may be detected usually by the formation of gas within the swellings upon the body, and the bubbles. thus developed will produce a crackling sound if the fingers are pressed over the affected area. Further, in blackleg the marked change in the affected musculature and the characteristic "rancidbutter" odor noted in cases of blackleg facilitate a differential diagrosis.

Hemorrhagic septicemia of swine, commonly termed swine plague, usually appears in the form of necrotic pleuropneumonia. The symptoms shown by the affected animal readily point to an attack of pneumonia, but the presence of roundworms in the lungs may cause identical manifestations. The long course of the latter diseaseshould be taken as an indication that the pig is not affected with swine plague. 
It is very difficult to distinguish swine plague from hog cholera. The two diseases may occur in the same animal. Should the outbreak seem to be but slightly contagious and not inclined to spread from the premises upon which it first appeared, it is probably not hog cholera, but, on the other hand, if it spreads rapidly throughout the neighborhood, it is undoubtedly due to hog-cholera infection. The presence of bipolar oval bacilli does not alone establish a diagnosis of swine plague, since those organisms are frequently found as secondary invaders in true cases of hog cholera, and again they are often present in the noses and throats of healthy swine.

\section{PREVENTION.}

Animals and fowls may be protected experimentally from contracting hemorrhagic septicemia through the use of bacterins. Cattle, sheep, swine, rabbits, and fowls, if treated with heated cultures of hemorrhagic septicemia germs obtained from animals or birds of the same species as that to which they themselves belong, will almost invariably become protected against injections of living cultures of the same germ, even though applied in comparatively large quantities. Tests made by this bureau have shown that the use of cultures from animals of another species often affords similar complete immunity. Sheep have been made immune from virulent cultures obtained from other sheep by the use of prepared cultures from cattle. Rabbits have been made resistant to hemorrhagic septicemia cultures, derived from a variety of different species of animals, by treatment with prepared cultures coming from animals of other species. They have been protected perfectly from inoculations with virulent cultures of the true Bacillus bovisepticus in doses four times as large as that required to produce fatal results in a rabbit receiving no previous treatment. Fowls have been protected from injections of deadly quantities of virulent fowl-cholera organisms by the use of prepared bouillon growths of the same germ, or by the use of strains of the fowl-cholera bacillus possessing but little virulence. However, when the colon or paratyphoid B group of organisms is found to be responsible for the deaths, the bacterins should include these germs.

As a result of these investigations, and of others reported in medical literature, it is customary, at the present time, in manufacturing bacterins for the protection of stock from attacks of hemorrhagic septicemia to use several cultures coming from a number of outbreaks of the disease for treating the same species of animals as that from which the cultures were obtained. For example, the bacterin for bovine hemorrhagic septicemia is being prepared from strains of the $B$. bovisepticus and not the $B$. suisepticus, or vice versa. 
In the October, 1916, issue of the American Sheep Breeder, Dr. W. H. Lytle, Oregon State Veterinarian, reports very satisfactory results from vaccination with an attenuated or weakened culture of the living organism. About 3,000 sheep were treated with the material. After 48 hours subsequent to the vaccination of the flocks only nine sheep were lost, although previous to inoculation several animals died each day. Bacterins made from the killed organisms of hemorrhagic septicemia have been used also with considerable success in Oregon bands of sheep.

\section{TREATMENT.}

In most cases treatment of a fully established case of hemorrhagic septicemia in an animal of any species is quite useless. All apparently well animals should be removed from those that are sick by placing them in separate, noninfected quarters. If new cases develop among them in a few days after their removal, the healthy ones remaining should be removed again to another locality. In that way the unaffected animals soon will be out of danger of further contamination, especially if their strength has been supported by an abundance of good feed and water during the period of their separation.

\section{DISINFECTION OF PREMISES.}

Premises usually become infected with hemorrhagic septicemia by stock cattle that have recently passed through some of the larger cattle markets. Owing to this method of infection the stables and yards may not be so completely contaminated as they would be if the disease had developed spontaneously. In any event, all stables, sheds, or yards that have contained infected animals should be disinfected. The interior of the stables, especially the mangers and manure trenches, should be washed with a disinfectant, such as liquor cresolis compositus (U. S. P.) or carbolic acid, 6 ounces to a gallon of water in either case. The yards may be disinfected by the applieation of a solution made of 5 ounces of copper sulphate to a gallon of water. The best means of applying disinfecting solutions is afforded by the use of a spray pump such as is used in the spraying of orchard trees. All refuse and material from the stable and barnyard should be removed to a place not accessible to cattle, sheep, or hogs. The manure should be spread on fields and plowed under. A plentiful supply of light and air should be provided for the contaminated stables. Open fields or pasture lands are cleansed rapidly by the action of sunlight upon them. 


\section{DEPARTMENT OF AGRICULTURE PUBLICATIONS RELATING TO DISEASES OF ANIMALS.}

\section{AVAILABLE FOR FREE DISTRIBUTION.}

Milk Fever and Its Treatment. (Farmers' Bulletin 206.)

The Tuberculin Test of Cattle for Tuberculosis. (Farmers' Bulletin 351.)

Rabies or Hydrophobia. (Farmers' Bulletin 449.)

'Tuberculosis. (Farmers' Bulletin 473.)

Methods of Disinfecting Stables. (Farmers' Bulletin 480.)

Methods of Exterminating the Texas-Fever Tick. (Farmers' Bulletin 498.)

'Texas or Tick Fever. (Farmers' Bulletin 569.)

Foot-and-Mouth Disease. (Farmers' Bulletin 666.)

Sheep Scab. (Farmers' Bulletin 713.)

Frevention of Losses of Stock From Poisonous Plants. (Farmers' Bulletin 720.)

'Tuberculosis of Hogs. (Farmers' Bulletin 781.)

Anthrax or Charbon. (Farmers' Bulletin 784.)

Contagious Abortion of Cattle. (Farmers' Bulletin 790.)

The Sheep Tick and Its Eradication by Dipping. (Farmers' Bulletin 798.)

Hog Cholera. (Farmers' Bulletin 834.)

Screw-Worms and Other Maggots Affecting Animals. (Farmers' Bulletin .857.)

Cattle Lice and How to Eradicate Thein. (Farmers' Bulletin 909.)

Important Poultry Diseases. (Farmers' Bulletin 957.)

Directions for the Use of Blackleg Vaccine. (Bureau of Animal Industry Circular 23, fourth revision.)

Blackleg; Its Nature, Cause, and Prevention. (Bureau of Animal Industry Circular 31, sixth revision.)

Actinomycosis, or Lumpy Jaw. (Bureau of Animal Industry Circular 96.)

The Contro' of Bovine Tuberculosis. (Bureau of Animal Industry Circular 175.)

Bighead in Sheep. (Leaflet A. I. 3.)

Glanders and Farcy. (Leaflet A. I. 13.)

FOR SALE BY THE SUPERINTENDENT OF DOCUMENTS, GOVERNMENT PRINTING OFFICE, WASHINGTON, D. C.

Immunization Tests With Glanders Vaccine. (Department Bulletin 70.) Price, 5 cents.

The Granular Venereal Disease and Abortion in Cattle. (Department Bulletin 106.) Price, 10 cents.

The Effect of the Cattle Tick Upon the Milk Production of Dairy Cows. (Department Bulletin 147.) Price, 5 cents.

Ophthalmic Mallein for the Diagnosis of Glandeis. (Department Bulletin 166.) Price, 5 cents.

The Dog as a Carrier of Parasites and Disease. (Department Bulletin 260.) Price, 5 cents.

Experiments in Vaccination Against Anthrax. (Department Bulletin 340.) Price, 5 cents. 
An Intrajermal Test for Bacterium Pullorum Infection in Fowls. (Department Bulletin 517.) Price, 5 cents.

The Control of Hog Cholera. (Department Bulletin 584.) Price, 5 cents.

Tuberculosis of the Food-Producing Animals. (Bureau of Animal Industry Bulletin 38.) 1906. Price, 40 cents.

Necrotic Stomatitis. (Bureau of Animal Industry Bulletin 67.) Price, 10 cents.

Ostecporosis or Bighead of the Horse. (Bureau of Animal Industry Circular 121.) Price, 5 ceuts.

Infectious Anemia or Swamp Fever of Horses. (Bureau of Animal Industry Circular 138.) Price, 5 cents.

Lip-and-Leg Ulceration of Sheep. (Bureau of Animal Industry Circular 160.) Price, 5 cents.

Diagnosis of Tuberculosis by Complement Fixation, With Special Reference to Bovine Tuberculosis. (In Journal of Agricultural Research, January 3, 1917.) A-26. Price, 10 cents.

Some Facts About Tuberculous Cattle. (Yearbook Separate 476.) Price, 5 cents.

Special Report on Diseases of Cattle. Price, \$1.

Special Report on Diseases of the Horse. Price, $\$ 1$. 
